\title{
Spontaneous rheumatoid fistula of the elbow joint
}

\author{
Gareth Huston ${ }^{1}$ and Raymond J. Newman ${ }^{2}$ \\ ${ }^{1}$ Seacroft Hospital, York Road, Leeds LS14 6UH and ${ }^{2}$ University Department of Orthopaedic Surgery, \\ Clinical Sciences Building, St James's University Hospital, Beckett Street, Leeds LS9 7TF, UK.
}

\begin{abstract}
Summary: A case of rheumatoid arthritis, whose presenting problem was a non-infective fistula of the elbow joint, is described. Penicillamine therapy was ineffective, but synovectomy led to healing.
\end{abstract}

\section{Introduction}

Joint fistulization is an uncommon feature of chronic rheumatoid arthritis and of published cases most have communicated with septic joints of sero-positive patients. Some infections have occurred primarily but most have followed prosthetic arthroplasty. ${ }^{1-4}$

Spontaneous fistulization of a non-infected rheumatoid joint is a rare occurrence and to our knowledge only six cases have been published ${ }^{1-3}$ involving mainly small joints of the feet and hands.

We wish to describe a patient without previous history of rheumatoid disease presenting primarily with a spontaneous fistula of a major joint together with the attempted medical and subsequent successful surgical treatment.

\section{Case report}

A 65 year old man without previous history of joint disease presented with a 7-month history of discharge from the lateral aspect of the left, nondominant, elbow. Three months previously he had noticed the development of a swelling which gradually enlarged, ruptured and subsequently discharged thin, occasionally blood-stained fluid.

Examination revealed $10^{\circ}$ fixed flexion deformity of both elbows, two discharging fistulae on the lateral aspect of the left elbow just distal to the lateral epicondyle (Figure 1) and a $3 \mathrm{~cm}$ diameter fleshy swelling over the lateral aspect of the right radial head. Apart from mild bilateral synovitis of the metacarpo-phalangeal joints the rest of the examination was normal.

The discharging fluid demonstrated fibrin clot and a few inflammatory cells. Initial culture grew Corynebacterium hofmannii but five subsquent cul-

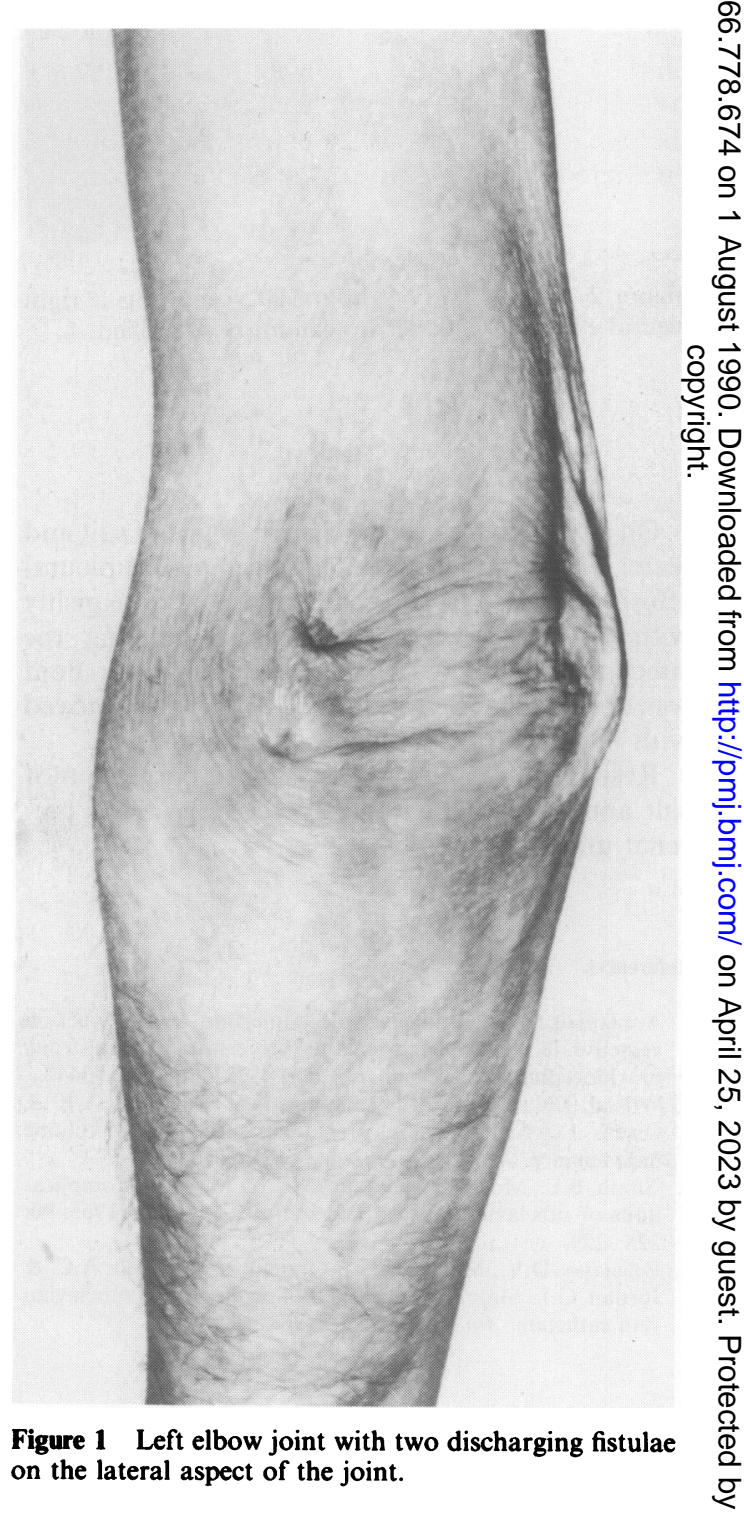


tures were sterile. Radiographs of the elbows showed the changes of erosive rheumatoid arthritis but with an air arthrogram on the left (Figure 2). Both hands and feet showed typical rheumatoid erosions. Plasma viscosity was 1.8, ANA was positive at $1 / 800$ and was homogenous and the latex test was negative. D-Pencillamine $375 \mathrm{mg}$ daily was prescribed for 5 months, nausea precluding higher doses. There was no significant effect on the discharge or the size of the swelling on the right side.

On the left side the fistula was excised and a limited synovectomy performed via the same small incision. On the right side the swelling was explored, found to be a rheumatoid nodule and excised together with a synovectomy of the elbow joint. Bacteriological culture of all excised tissue was sterile. The wound on the right healed primarily but on the left side the fistula recurred within 2 weeks. A synovectomy as complete as possible was therefore performed via an enlarged incision and facilitated by excision of the radial head. The

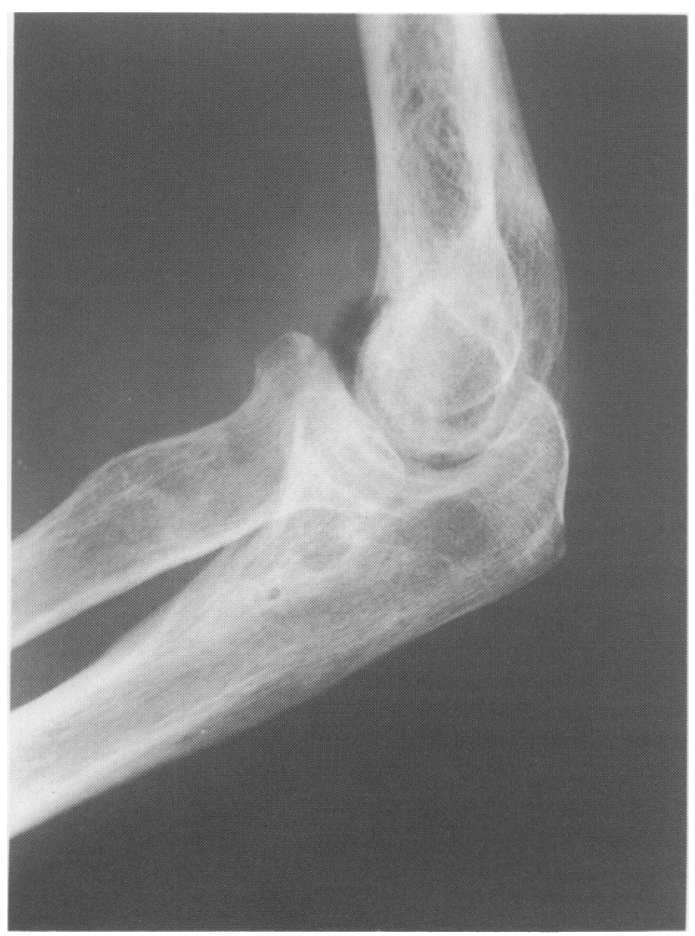

Figure 2 Radiograph of the left elbow showing osteopenia, erosive arthritis and an air arthrogram communicating with the lateral aspect of the joint. wound remained dry post-operatively and healed primarily. Culture of the excised synovium was again sterile. Histology of the tissue removed from both elbows was identical and was that of rheumatoid nodules. On the left side the fistula passed through the nodule.

\section{Discussion}

Joint fistulization is a recognized but uncommon complication of sero-positive rheumatoid arthritis with most cases related to infection either low grade $^{1-3}$ or suppurative. ${ }^{4}$ Those arising from the metatarso-phalangeal joints require excison forefoot arthroplasty together with synovectomy and excision of associated rheumatoid cysts for their cure. ${ }^{1,3,5}$ Those secondary to prosthetic arthroplasty may require extensive bony surgery and debridement. ${ }^{6}$ The only reported cure of a non-infective rheumatoid fistula by soft tissue excision related to one communicating with a dissecting popliteal cyst rather than a true joint. ${ }^{3}$

The present case is the first description of a fistula involving a major joint of the upper limb without previous history of rheumatoid arthritis. Though one culture of the discharging fluid was positive for a rare organism five subsequent cultures were sterile suggesting that the initial organism was a contaminant. The negative latex test was surprising and might represent a case of so-called 'hidden' rheumatoid factor, ${ }^{7,8}$ as the presence of typical rheumatoid nodules as well as erosions precludes the diagnosis of typical seronegative 'definite' rheumatoid arthritis.

The prescription of anti-rheumatic chemotherapy was an attempt to heal the fistula by decreasing the production of synovial fluid. Failure of this form of therapy reflects the inefficiency of D-penicillamine in inhibiting a florid synovitis at the tolerated dose. Since the histology was identical on both sides it is interesting to speculate whether a fistula would have developed in the right elbow had surgical intervention not ensued. Spontaneous fistula formation is an uncommon complication of non-infected rheumatoid arthritis and it is suggested that it be treated surgically rather than medically.

\section{Acknowledgements}

We wish to thank Mesdames Sue Briggs and Esther Newman for secretarial support. 


\section{References}

1. Bywaters, E.G.L. Fistulous rheumatism. A manifestation of rheumatoid arthritis. Ann Rheum Dis 1953, 12: 114-121.

2. Rosin, A.J. \& Toghill, P.J. Fistulous rheumatism. An unusual complication of rheumatoid arthritis. Postgrad Med J 1963, 39: 96-98.

3. Shapiro, R.F., Reswick, D., Castles, J.J., D'Ambrosia, R., Lipscomb, P.R. \& Niwayama, G. Fistulization of rheumatoid joints. Spectrum of identifiable syndromes. Ann Rheum Dis 1975, 34: 489-498.

4. Kellgren, J.H., Ball, J., Fairbrother, R.W. \& Barnes, K.L Suppurative arthritis complicating rheumatoid arthritis. Lancet 1958, i: 1193.
5. Newman, R.J. \& Fitton, J.M. Conservation of metatarsal heads in surgery of rheumatoid arthritis of the forefoot. Acta Orthop Scand 1983, 54: 417-421.

6. Elson, R.A. General technique in revision arthoplasty. In: Elson, R.A. (ed.) Revision Arthroplasty 2. Franklin Scientific Publications, London, 1983, pp. 69-72.

7. Lindstrom, F.D. Hidden rheumatoid factor in active seronegative rheumatoid arthritis. Scand J Rheumatol 1973, 2: 133-135.

8. Allen, J. \& Kunkel, H.G. Hidden rheumatoid factors with specificity for native gamma globulins. Arthritis Rheum 1966, 9: $758-767$. 